\title{
Initial data on protective containment for safety analysis of NPP with WWER-1000 Preparation
}

\author{
Oleg Gubeladze ${ }^{1}$, Avtandil Gubeladze ${ }^{1}$, Larisa Kiril'chik $^{1}$ \\ ${ }^{1}$ Don State Technical University, Rostov-on-Don, Russia
}

\begin{abstract}
In the presented article the nuclear safety issues with VVER1000 reactor are considered. The study element is the protective containment (PC), the most important function of which is localization and retention of radioactive substances within the accident localization zone. The example of possible unregulated destructive forcing (UDF) on the PC for the construction and installation work period is given.
\end{abstract}

One of the basic concepts of nuclear power plants with WWER safety is the concept of defense-in-depth, which includes the installation of successive physical barriers: fuel matrix and fuel element cladding; coolant contour; protective containment (PC). In order to implement the concept it is necessary to create several layers of protection "in depth" [1]. The following levels are of particular interest: maintaining ready components and systems of nuclear power plants equipment, which are important for the safety of the facility (equipment redundancy, technical condition diagnostics, malfunctions and failures elimination, qualitative carrying out of preventive maintenance); preventing the transition process parameters exceed the safe operation.

The most important function of PC under unfavorable circumstances is considered to be the location and retention of radioactive substances within the accident localization zone. The effectiveness of the containment protection functions implementation is strongly influenced by its successful resistance to unregulated destructive forcing (UDF).

The procedure for the assessment of the technical state of PC for the preparation of input data for the PSA includes: the technical documentation analysis, visual inspection, tool inspection, results analysis, testing calculations performance. As an example, consider a survey of containment during the construction of the power generating unit of a nuclear power plant. Implementation of instrumental examination from the mark $13.2 \mathrm{~m}$ to $55.6 \mathrm{~m}$ was carried out by non-destructive testing methods. To control the concrete strength characteristics the shock pulse method based device was used. Determination of the protective layer of reinforcement was carried out by the device based on magnetic method of nondestructive testing. Cracks parameters were determined by the depth of the cracks with an ultrasonic device; by the crack opening width with a measuring microscope. Control of the spatial position of the containment (rainfall, rolls, rotations) was performed by geodetic measurement methods.

*Oleg Gubeladze: buba26021966@yandex.ru 
We distinguish PC as an element for further study. The object $S$ can be represented in the form of the model (Fig. 1), which has the following characteristics: X - input effect $\left(x_{a} \in X, a=\overline{1, N_{x}}\right) ; H$ - internal system parameters $\left(h_{l} \in H, l=\overline{1, N_{h}}\right) ; \quad Y$ - output parameters $\left(y_{l} \in Y, b=\overline{1, N_{y}}\right) ; V$ - a set of external disturbances $\left(v_{l} \in V, k=\overline{1, N_{v}}\right)$.

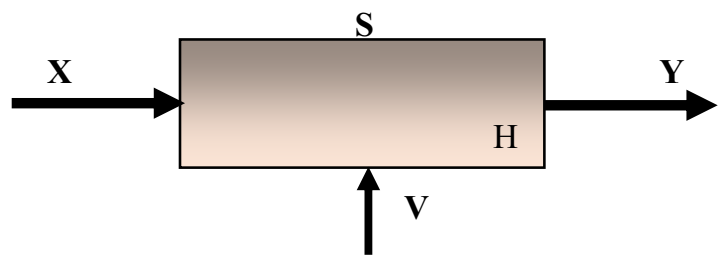

Fig. 1. The model of the object.

In vector form

$$
\left\{\begin{array}{l}
\vec{x}(t)=x_{1}(t), x_{2}(t), \ldots, x_{N_{x}}(t) ; \\
\vec{h}(t)=h_{1}(t), h_{2}(t), \ldots, h_{N_{h}}(t) ; \\
\vec{v}(t)=v_{1}(t), v_{2}(t), \ldots, v_{N_{v}}(t) ; \\
\vec{y}(t)=y_{1}(t), y_{2}(t), \ldots, y_{N_{y}}(t),
\end{array}\right.
$$

The mathematical model of the object is given by

$$
\vec{y}(t)=F_{s}(\vec{x}, \vec{h}, \vec{v})
$$

where $F s$ - transformation operator.

In case there is a set of external disturbances UDF ( $V U D F \cap V, v d^{\prime} \varepsilon V U D F, d=1, N d$ ) it may cause the accident situation (AS), in case of the development of which outcomes $G$ are expected (Fig. 2), where $g_{j} \varepsilon G$.

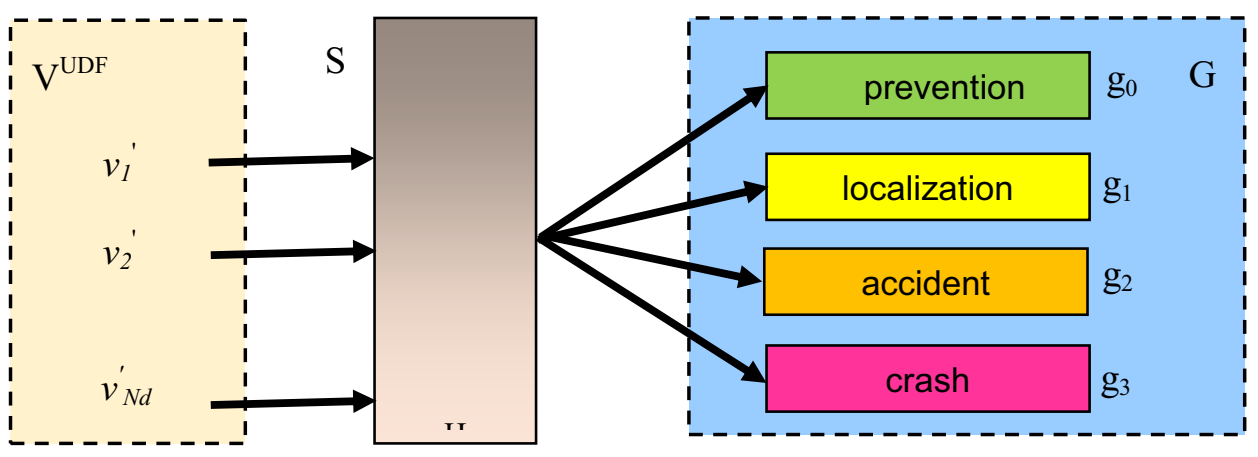

Fig.2.

Outcome criteria are characterized by $\mathrm{K}(\mathrm{g} j)$, in this case, preference $\mathrm{G}$ can be described by a single $\mathrm{K}$ criterion, i.e. the outcome $\mathrm{g} 0$ is preferable to others

$$
K\left(g_{0}\right) \succ K\left(g_{j}\right), j=1,2,3 \text {. }
$$

In order to prevent the AS emergence or further development some protection object $\mathrm{S}$ measures are implemented. These measures implementation depends on the efficiency of 
both individual components and the entire protection system (PS) [2]. Ways to implement security measures will be called $U$ strategies ( $u m \varepsilon U, m=1, N u$ ).

In case the problem is characterized by the presence of random factors with known distribution laws (equipment failure, random «demand", etc.) any strategy of $U$ variety can be associated with the distribution of outcomes multifold. In this case, $G$ is a finite set, so for the strategy $U m$ under influence of $v d$ ' the occurrence of each outcome $g j$ can be probably determined as $P j\left(u m, v d^{\prime}\right)$. Criterion characterizing outcomes is a random function $K(U m)$. The distribution $K(U m)$ is known for any chosen strategy. Having specified the number $\mathrm{Z}$, it is possible to determine the probability that the $K(U m)$ value will not be less than $Z$

$$
P\left(\widetilde{K}\left(u_{m}\right) \geq Z\right)=\gamma\left(z, u_{m}\right)
$$

According to the principle of probabilistic guaranteed result the required level is given and numerical function $K^{z}(u)=\gamma(z, u)$ is defined, i.e the optimal strategy is defined as determining $\max K^{z}(u)$, where $u \varepsilon U$.

However, the task is complicated by the presence of uncertainty. It is connected with the remoteness of the direct application PS stage, uncertainty of the moment of its use, system characteristics (at the time of UDF appearance, capable of causing AS), as well as the future using conditions (the combination of UDF and their parameters). Protective containment, being part of the PS in its turn is also influenced by UDF. This can be applied not only to direct impacts at the time of AS but also UDF in the preceding period of power generating unit (construction, testing, operation). As an example (Fig. 3) shows the possible UDF on PC. In order to eliminate uncertainty in power generating unit probabilistic safety analysis (PSA) it is necessary to get as much as possible complete and accurate data concerning the parameters of PS elements $[3,4]$ achieved in the implementation of design solutions in the construction of containment.

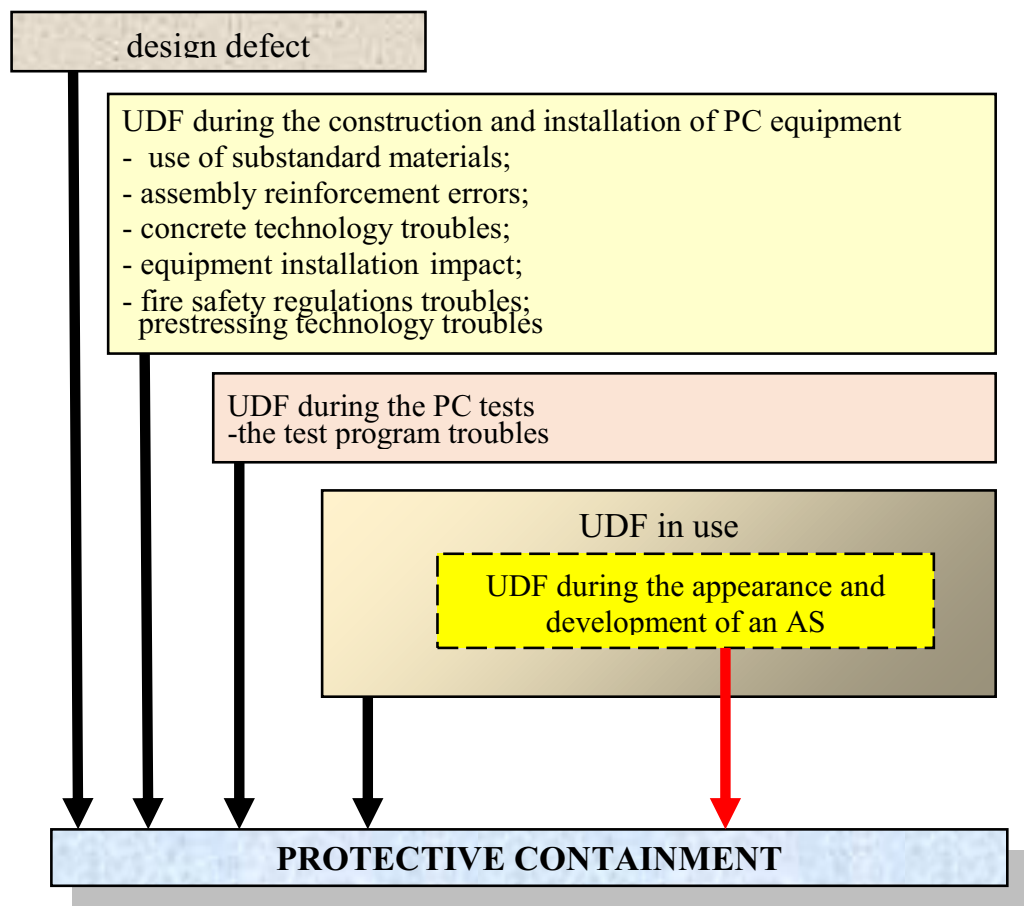

Fig.3. The possible UDF on PC 
This approach allowed us to identify an impact of materials handling systems for reactor compartments in the building construction PC during construction and installation works [5]. Within marks 46,00 and $49.00 \mathrm{~m}$, i.e. on the horizon polar crane support brackets on the outer side some cracks were detected, substantially uniformly distributed across the circumference of the shell (Fig. 4).
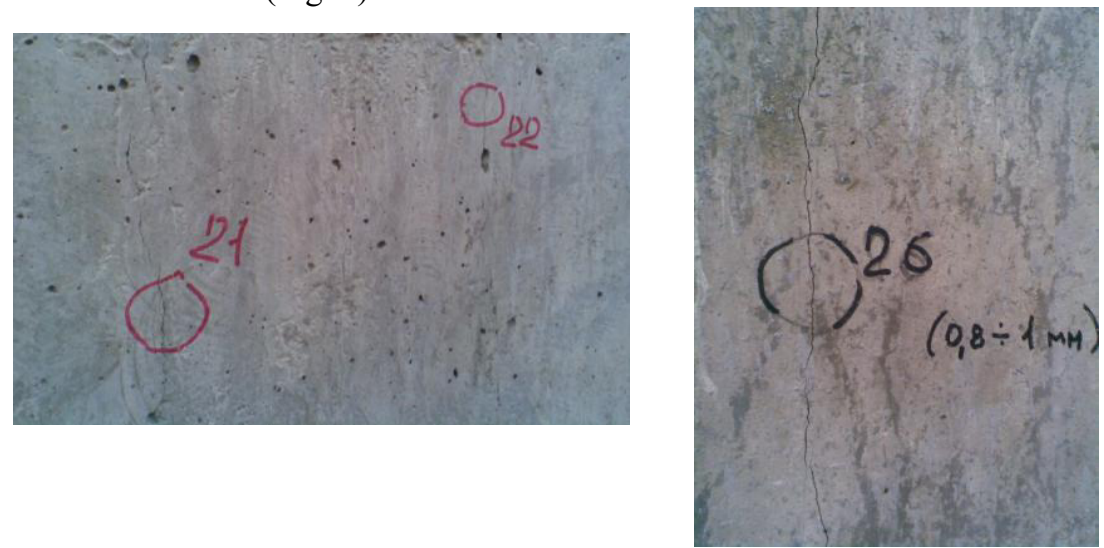

Fig.4. Cracks on the outer surface of PC.

After the crane mounting depending on the availability of construction combinations of different integral stiffness of shell structure are formed, which eventually leads to a different deformation in the direction of a building structure tier. Till the full construction PC readiness, the crane goes through several stages of testing. Installation of primary circuit equipment is also performed on a non stressed shell. In each case there occurs a corresponding deformation of the shell, which also leads to the cracks formation on the outer side of the shell wall. In the construction draft of PC a special requirement is formulated; that is shrinkage cracks with opening of $0.3 \mathrm{~mm}$ are accepted. In reality, these values are exceeded several times, and the depth of the crack may reach $160 \mathrm{~mm}$.

For mandatory performance of instrumental control of PC works in the required amount, it is necessary to develop a package of universal standard programs.

\section{References}

1. V. Ostreykovsky, Y. Shvyryaev. Safety of nuclear power stations. Probabilistic analysis, 352 (2008)

2. O. Gubeladze. Universities' News. North Caucasus region. Tech. science. Special Issue, 127 (2008)

3. Inspection of building constructions. Organization standard of self regulated organization 60542948 00005-2012

4. Nuclear facilities. Inspection of building constructions. Organization and rules for the survey of building designs of nuclear power plants. Organization standard 95 105-2013

5. Y. Pimshin, E. Klyushin, O. Gubeladze and others. Global Nuclear Safety, 2, 33 (2016) 